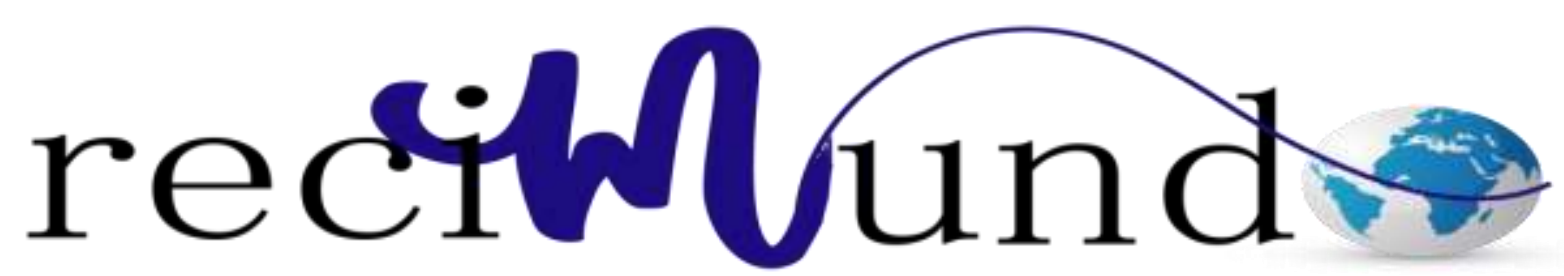

Revista Cientifica Mundo de la Investigación y el Conocimiento

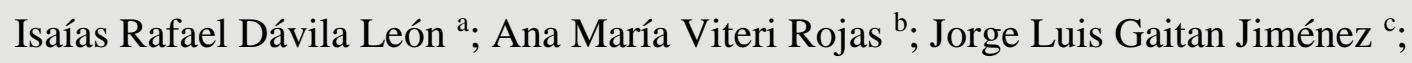

Karina Johana Cevallos Cárdenas d; Katherine Angélica Sánchez Alcántara e

Prevalencia y factores de riesgo de hernias incisionales en adultos en el Hospital

Abel Gilbert Pontón desde enero del 2014 a diciembre del 2017

Prevalence and risk factors of incisional hernias in adults at the Abel Gilbert

Pontón Hospital from January 2014 to December 2017

Revista Científica Mundo de la Investigación y el Conocimiento. Vol. 3 núm.2,

abril, ISSN: 2588-073X, 2019, pp. 251-263

DOI: $\underline{10.26820 / \text { recimundo/3.(2).abril.2019.251-263 }}$

URL: http://www.recimundo.com/index.php/es/article/view/444

Código UNESCO: 3205 Medicina Interna

Tipo de Investigación: Artículo de Revisión

Editorial Saberes del Conocimiento

Recibido: 15/01/2019

Aceptado: 18/02/2019

Publicado: 30/04/2019

Correspondencia: isaiasdavida.1eon@gmail.com
a. Médico General; isaiasdavida.leon@gmail.com
b. Especialista en Cirugía General; Doctora en Medicina y Cirugía; Docente de Cirugía de la Universidad de Guayaquil; anyviten031@ hotmail.com
c. Médico General; jorge gaitan14@hotmail.com
d. Magister en Atención Primaria y Clínica Infantil; Médico; kannacevallos75@ hotmail.com
e. Médico. 


\section{Prevalencia y factores de riesgo de hernias incisionales en adultos en el Hospital Abel Gilbert Pontón desde enero del 2014 a diciembre del 2017}

Vol. 3, núm. 2., (2019)

Isaías Rafael Dávila León; Ana María Viteri Rojas; Jorge Luis Gaitan Jiménez; Karina Johana Cevallos Cárdenas; Katherine Angélica Sánchez Alcántara

\section{RESUMEN}

Una de las primeras patologías que se presentan más salta a la mente, son las hernias. Una enfermedad cuya presentación en las consultas de primer nivel e incluso en las emergencias son muchísimo más comunes de lo que se tiene documentado. Una patología que obliga al cirujano, y por qué no a médicos generales y estudiantes, un conocimiento adecuado y amplio sobre la anatomía de la pared abdominal y la fisiopatología herniaria. El tratamiento requiere un abordaje amplio y completo que permitan anticipar cualquier posible complicación transquirúrgica o postquirúrgica, tomando en cuenta factores de riesgo sistémicos como edad, obesidad, diabetes, trastornos hepáticos, antecedentes de cirugías previas. Es importante asimismo señalar la importancia de la técnica quirúrgica y el material de sutura a usados durante la cirugía. En los últimos años el avance en las técnicas quirúrgicas en la patología herniaria ha sobrepasado en sobremanera al resto siendo quizás, únicamente la cirugía bariátrica la que haya tenido un avance medianamente comparable.

Palabras Claves: Hernias Incisionales; Factores de Riesgos; Adultos; Cirugía. 


\title{
Prevalencia y factores de riesgo de hernias incisionales en adultos en el Hospital Abel Gilbert Pontón desde enero del 2014 a diciembre del 2017
}

Vol. 3, núm. 2., (2019)

Isaías Rafael Dávila León; Ana María Viteri Rojas; Jorge Luis Gaitan Jiménez; Karina Johana

Cevallos Cárdenas; Angélica Katherine Sánchez Alcántara

\begin{abstract}
In the professional field and in this case, the academic, when dealing with the specialty of general surgery, one of the first pathologies that come to mind of any student or professional, are hernias. A disease whose presentation in first level consultations and even in emergencies is much more common than what is documented. A pathology that forces the surgeon, and why not general practitioners and students, adequate and comprehensive knowledge about the anatomy of the abdominal wall and hernia physiopathology. The treatment requires a broad and complete approach to anticipate any possible transurgical or postsurgical complication, taking into account systemic risk factors such as age, obesity, diabetes, liver disorders, and history of previous surgeries. It is also important to note the importance of the surgical technique and the suture material used during surgery. In recent years the advance in surgical techniques in hernia pathology has greatly exceeded the rest being perhaps, only bariatric surgery that has had a fairly comparable progress.
\end{abstract}

Key Words: Incisional Hernias; Risk Factors; Adults; Surgery. 


\section{Prevalencia y factores de riesgo de hernias incisionales en adultos en el Hospital Abel Gilbert Pontón desde enero del 2014 a diciembre del 2017}

Vol. 3, núm. 2., (2019)

Isaías Rafael Dávila León; Ana María Viteri Rojas; Jorge Luis Gaitan Jiménez; Karina Johana Cevallos Cárdenas; Katherine Angélica Sánchez Alcántara

\section{Introducción.}

Las hernias incisionales son una complicación muy común en la cirugía abdominal y una causa de morbilidad muy frecuente en pacientes intervenidos quirúrgicamente. A pesar de que es problema muy frecuente no se tiene un consenso general con respecto a su tratamiento. La presentación de esta complicación en adultos es cada vez más frecuente, factores de riesgo como gran cantidad de cirugías previas, características particulares de los pacientes (estilo de vida trabajo), comorbilidades (Diabetes, obesidad, desnutrición, alcoholismo, tabaquismo), uso de técnica quirúrgica o materiales no acorde a las necesidades del paciente originan la creciente incidencia de esta patología, pero asimismo se desconoce el alcance real de la misma en nuestro medio. $^{1-3}$

Bajo este antecedente se formula una interrogante que forma parte de la problemática del estudio que es: ¿Cuáles son los factores de riesgo asociados con el aumento de la prevalencia del diagnóstico de hernias incisionales en adultos mayores en el servicio de cirugía en el Hospital Abel Gilbert Pontón?

En este proyecto investigativo se plantea conocer de una manera real el número de pacientes adultos mayores diagnosticados con hernia incisional y cuál es su asociación con los distintos factores de riesgo presentes en estos pacientes. Para esto se hará una revisión bibliográfica y estadística de las historias clínicas de los pacientes con este diagnóstico en el Hospital Abel Gilbert Pontón, por lo cual es un proyecto viable. Siendo el objetivo general: Conocer mediante un estudio analítico la prevalencia de casos de hernias incisionales en pacientes adultos mayores. ${ }^{4-10}$ 


\section{Prevalencia y factores de riesgo de hernias incisionales en adultos en el Hospital Abel Gilbert Pontón desde enero del 2014 a diciembre del 2017}

Vol. 3, núm. 2., (2019)

Isaías Rafael Dávila León; Ana María Viteri Rojas; Jorge Luis Gaitan Jiménez; Karina Johana

Cevallos Cárdenas; Angélica Katherine Sánchez Alcántara

Los datos de prevalencia e incidencia no dan ninguna indicación sobre la demanda real o potencial de cirugía de hernia. A pesar de que incompleta y sujeta a muchos errores en la interpretación, fuentes de datos de Reino Unido que se relacionan con la necesidad de cirugía de hernia incluye los datos de consulta hospitalaria del Hospital Inglés (HIPE), 1975-1985; los datos del Sistema de Episodios del Hospital Inglés (HES),1989/1990; y datos sobre la actividad quirúrgica en Hospitales Independientes en el Servicio Nacional de Salud (NHS) de la localidad y encuestas nacionales. ${ }^{5-8}$

La anatomía de la pared abdominal ha sido bien documentada en varios textos de referencia anatómicos estándar. Ciertos procesos patológicos pueden, en ocasiones, distorsionar la anatomía subyacente, y el cirujano debe ser consciente y tener en cuenta estas alteraciones para garantizar resultado exitoso de la cirugía de hernia. De manera óptima, el cirujano debe adaptar cada operación a la anatomía específica encontrada en el paciente individual. ${ }^{10-14}$

Este reexamen detallado de la anatomía de la pared abdominal (tanto topográfico como funcional) ha resultado en una mejora significativa en nuestra comprensión del desarrollo hernias y también ha resultado en la generación de muchos consejos prácticos para cirujanos en el manejo quirúrgico de hernias, en particular cuando se enfrentan a formas variantes de hernia que divergen de las descripciones estándar La región abdominal se encuentra entre la región torácica por arriba y la región pélvica por debajo. Los límites de la región torácica son claros, el músculo diafragma en la parte superior, sin embargo entre la región abdominal y la pélvica no existen límites anatómicos reales, y existe una continuidad entre ambas, por lo que se considera a la cavidad abdominal y pelviana como una sola, la cavidad abdominopélvica. 


\section{Prevalencia y factores de riesgo de hernias incisionales en adultos en el Hospital Abel Gilbert Pontón desde enero del 2014 a diciembre del 2017}

Vol. 3, núm. 2., (2019)

Isaías Rafael Dávila León; Ana María Viteri Rojas; Jorge Luis Gaitan Jiménez; Karina Johana Cevallos Cárdenas; Katherine Angélica Sánchez Alcántara

Se conoce como hernia a la protrusión de un tejido, órgano u otra estructura anatómica a través del tejido muscular o membrana en los cuales es contenido normalmente o a través de un orificio anatómicamente constituido pero que debido a diversas situaciones se ha dilatado. Las hernias pueden ser internas, externas, congénitas o adquiridas.

La hernia abdominal es la salida de estructuras intraabdominales a través de la pared abdominal. Para su estudio la dividimos en dos partes: una apertura en la pared (defecto herniario) y un saco herniario consistente en peritoneo y contenido abdominal. Estas hernias abarcan las hernias de la ingle (hernia femoral, hernia inguinal) y la hernia ventral.

Se la define como una hernia inguinal que aparece inferior al ligamento inguinal y hacia la parte interna de la vena y arteria femorales. El saco herniario femoral tiene un cuello de tamaño pequeño, pero puede crecer de manera considerable cuando penetra en el tejido celular subcutáneo del muslo. Es causada por defectos en la pared abdominal. Son hernias primarias que aparecen en la pared abdominal y son independientes de las hernias inguinales. Se trata de una hernia ocasionada por un defecto de la pared abdominal anterior debida a alteraciones de la línea media, incisiones previas o aumento de la presión intraabdominal.

Las hernias umbilicales se forman a través de defectos en el cierre del orificio abdominal donde el cordón umbilical emerge después de que el saco celómico se oblitera. Durante la tercera semana de desarrollo, la piel y las cubiertas fasciales de la pared abdominal cubren los contenidos intraabdominales. Las hernias umbilicales se forman a través de defectos en el cierre del orificio abdominal donde el cordón umbilical emerge después de que el saco celómico se 


\section{Prevalencia y factores de riesgo de hernias incisionales en adultos en el Hospital Abel Gilbert Pontón desde enero del 2014 a diciembre del 2017}

Vol. 3, núm. 2., (2019)

Isaías Rafael Dávila León; Ana María Viteri Rojas; Jorge Luis Gaitan Jiménez; Karina Johana

Cevallos Cárdenas; Angélica Katherine Sánchez Alcántara

oblitera. Durante la tercera semana de desarrollo, la piel y las cubiertas fasciales de la pared abdominal cubren los contenidos intraabdominales.

La hernia incisional es iatrogénica y corresponde a la protrusión de contenido abdominal a través de una incisión quirúrgica previa, y su incidencia ha aumentado con el incremento de la intervención quirúrgica abdominal. Una hernia incisional es el ejemplo más perfecto de una "variable cirujano dependiente". Estas revelan un evidente fracaso al momento de la reparación de la pared abdominal. La intervención laparoscópica ha permitido la disminución de la incidencia de hernia incisional en sitios de implantación de trócares Además representa un importante factor de morbilidad incluyendo encarceramiento en un 7 a $14 \%$ de los casos y estrangulamiento en un $2.5 \%$.

Excepto por episodios de obstrucción aguda y estrangulación, las complicaciones per se de tener una hernia son raras. Como consecuencia, la descripción de las complicaciones generales se basa en casos individuales o series de casos, a menudo de publicaciones de otra manera considerada histórica. Mientras la edad de las publicaciones por sí solo puede no ser un problema, la aplicabilidad a la población actual de pacientes y conocimiento médico actual y la tecnología puede ser cuestionable en algunos casos. 
Prevalencia y factores de riesgo de hernias incisionales en adultos en el Hospital Abel Gilbert Pontón desde enero del 2014 a diciembre del 2017

Vol. 3, núm. 2., (2019)

Isaías Rafael Dávila León; Ana María Viteri Rojas; Jorge Luis Gaitan Jiménez; Karina Johana Cevallos Cárdenas; Katherine Angélica Sánchez Alcántara

Desarrollo (Datos Estadísticos)

Tabla 1: Prevalencia de pacientes con hernias incisionales

\begin{tabular}{|c|c|}
\hline Hombres & 60 \\
\hline Mujeres & 147 \\
\hline TOTAL & $\mathbf{2 0 7}$ \\
\hline
\end{tabular}

Elaboración: Autores

Tabla 2: Distribución de prevalencia por años

\begin{tabular}{|c|c|}
\hline AÑO 2014 & 86 \\
\hline AÑ 2015 & 52 \\
\hline AÑO 2016 & 23 \\
\hline AÑO 2017 & 46 \\
\hline TOTAL & $\mathbf{2 0 7}$ \\
\hline
\end{tabular}

Elaboración: Autores

Tabla 3: Factores de riesgos. Pacientes que presentaron sólo uno

\begin{tabular}{|l|r|}
\hline HTA & 10 \\
\hline DIABETES & 4 \\
\hline CIRROSIS & 1 \\
\hline OBESIDAD & 15 \\
\hline CIRUGIAS GINECOLOGICAS PREVIAS & 28 \\
\hline TABAQUISMO & 2 \\
\hline TOTAL & 60 \\
\hline
\end{tabular}

Elaboración: Autores

Revista Científica Mundo de la Investigación y el Conocimiento. 3 (2). pp. 251-263 


\section{Prevalencia y factores de riesgo de hernias incisionales en adultos en el Hospital Abel Gilbert Pontón desde enero del 2014 a diciembre del 2017}

Vol. 3, núm. 2., (2019)

Isaías Rafael Dávila León; Ana María Viteri Rojas; Jorge Luis Gaitan Jiménez; Karina Johana

Cevallos Cárdenas; Angélica Katherine Sánchez Alcántara

Tabla 4: Asociaciones de factores de riesgo más frecuentes

\begin{tabular}{|l|r|}
\hline HTA MÁS OBESIDAD & 18 \\
\hline OBESIDAD MÁS CIRUGIAS GINECOLOGICAS PREVIAS & 45 \\
\hline HTA MÁS CIRUGÍAS GINECOLÓGICAS PREVIAS & 14 \\
\hline OTRAS ASOCIACIONES & 8 \\
\hline PACIENTES CON MÁS DE 2 FACTORES DE RIESGO & 34 \\
\hline TOTAL & 119 \\
\hline
\end{tabular}

Elaboración: Autores

Tabla 5: Prevalencia de presentación de hernias incisionales según la edad

\begin{tabular}{|c|c|}
\hline 15 a 30 años & 10 \\
\hline 31 a 45 años & 44 \\
\hline 46 a 60 años & 68 \\
\hline Mayores de 60 años & 85 \\
\hline TOTAL & $\mathbf{2 0 7}$ \\
\hline
\end{tabular}

Elaboración: Autores

\section{Resultados.}

La demostración de pequeñas hernias incisionales puede ser muy difícil. Pacientes con pequeñas salidas de grasa extraperitoneal y un saco peritoneal pequeño pueden que los mismos se quejen del bulto blando, que no siempre está presente, pero que causa Dolor localizado bastante severo cuando está presente. Físico examen del paciente supino y relajado por lo general revela la causa $\mathrm{El}$ examen de ultrasonido es útil prueba de diagnóstico y a menudo revelará un defecto impalpable, particularmente en el paciente obeso. Sin embargo, el examen ecográfico de la pared abdominal depende de un intérprete experto. 


\section{Prevalencia y factores de riesgo de hernias incisionales en adultos en el Hospital Abel Gilbert Pontón desde enero del 2014 a diciembre del 2017}

Vol. 3, núm. 2., (2019)

Isaías Rafael Dávila León; Ana María Viteri Rojas; Jorge Luis Gaitan Jiménez; Karina Johana Cevallos Cárdenas; Katherine Angélica Sánchez Alcántara

A veces es difícil diferenciar entre una hernia y la grasa subcutánea o el intestino delgado en la hernia. En la mayoría de las situaciones y particularmente para las masivas y complejas hernias incisionales, la tomografía computarizada puede ser mucho más eficiente y precisa para definir el defecto y la planificar la preparación preoperatoria del paciente y la cirugía a elegir.

La tomografía computarizada es particularmente útil en la obesidad y en pacientes con cicatrices extensas de laparotomía, ya que define el contenido del saco especialmente si las hernias son clínicamente ocultas. Además, se debe realizar un diferencial con otras enfermedades como hematoma, absceso y neoplasia.

Los factores causales importantes incluyen la sepsis $(60 \%$ de pacientes que desarrollan una hernia incisional en el primer año después de la cirugía han tenido una infección significativa de la herida), la colocación de tubos de drenaje a través de la incisión original, una operación previa a través de la misma incisión dentro de 6 meses, el cierre inicial con catgut solo ("métodos ineptos de sutura"), esteroides y otras terapias inmunosupresoras y enfermedad intestinal inflamatoria.

La obesidad es un importante factor de riesgo tanto por la ocurrencia de la hernia original incisional y para la probabilidad de recurrencia de la hernia después de la reparación. La dehiscencia temprana de la herida es frecuentemente seguida de una hernia incisional. Estas hernias pueden estar relacionadas con el efecto de aserrado de suturas no absorbibles en la aponeurosis. Los factores menos importantes incluyen la edad y género, anemia, desnutrición, hipoproteinemia, diabetes, tipo de incisión, obstrucción intestinal postoperatoria, e infección postoperatoria del pecho. 


\section{Prevalencia y factores de riesgo de hernias incisionales en adultos en el Hospital Abel Gilbert Pontón desde enero del 2014 a diciembre del 2017}

Vol. 3, núm. 2., (2019)

Isaías Rafael Dávila León; Ana María Viteri Rojas; Jorge Luis Gaitan Jiménez; Karina Johana Cevallos Cárdenas; Angélica Katherine Sánchez Alcántara

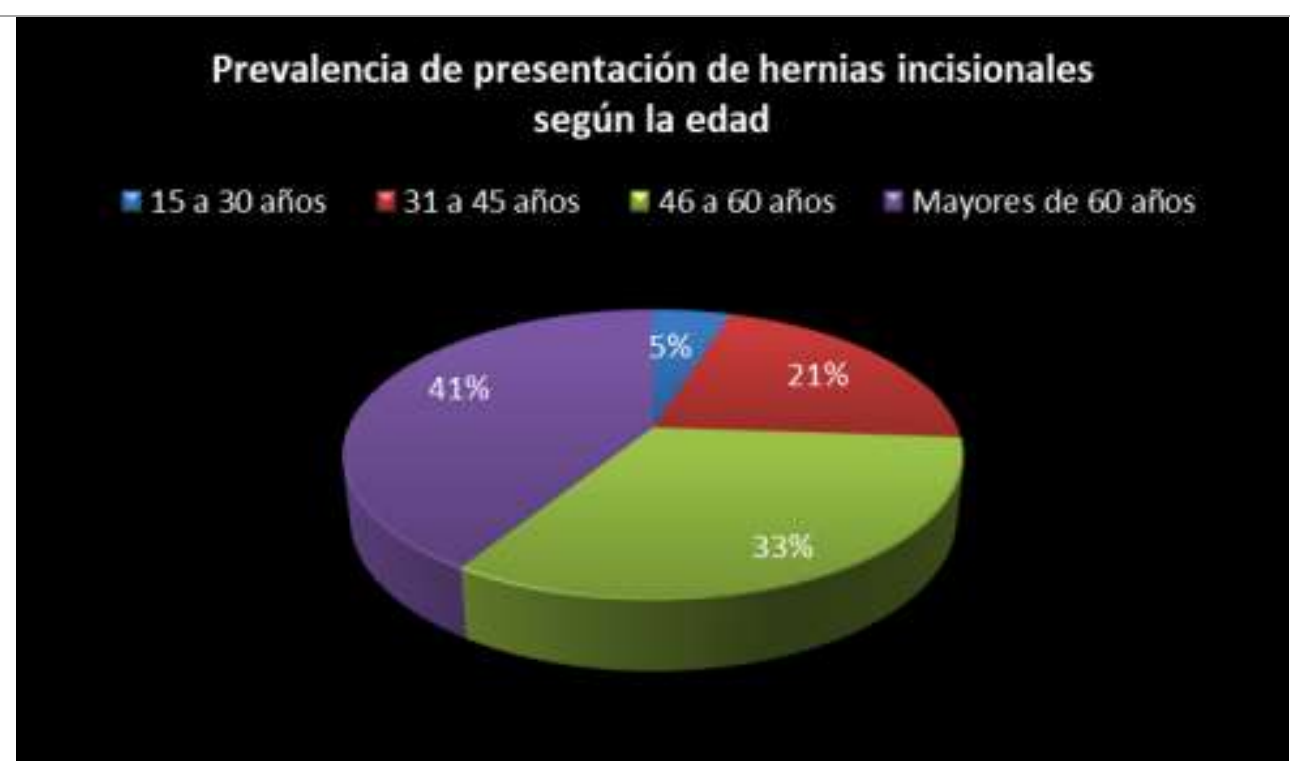

Figura 1: Prevalencia de presentación de hernias incisionales según la edad

\section{Conclusiones.}

Durante el desarrollo de este trabajo investigativo y basándose en la recopilación de los datos de historias clínicas y el registro de la base de datos del Hospital Abel Gilbert Pontón se obtuvieron los siguientes resultados:

[1] Entre enero del 2014 y diciembre del 2017 se registraron un total de 207 casos de hernias incisionales en el Hospital Abel Gilbert Pontón.

[2] La mayor prevalencia de hernias incisionales entre enero del 2014 y diciembre del 2017 se presentó en las pacientes de sexo femenino. 


\section{Prevalencia y factores de riesgo de hernias incisionales en adultos en el}

Hospital Abel Gilbert Pontón desde enero del 2014 a diciembre del 2017

Vol. 3, núm. 2., (2019)

Isaías Rafael Dávila León; Ana María Viteri Rojas; Jorge Luis Gaitan Jiménez; Karina Johana Cevallos Cárdenas; Katherine Angélica Sánchez Alcántara

[3] El factor de riesgo más comúnmente asociado a la presentación de hernias incisionales son aquellas pacientes con un historial de cirugías ginecológicas anteriores con un total de 102 pacientes.

[4] El 2do factor de riesgo más comúnmente asociado a la presentación de hernias incisionales fue la obesidad con un total de 94 pacientes.

[5] La asociación de factores de riesgo más común que se presentó durante los 3 años evaluados fue la Obesidad más Historial de cirugías ginecológicas previas con un total de 45 pacientes.

[6] Los pacientes que llegaron a tener más de un solo factor de riesgo fueron un total de 119 pacientes.

[7] El año donde hubo una mayor prevalencia de presentación de hernias incisionales fue el año 2014 donde se registraron 86 casos, mientras el año con menor prevalencia del estudio fue el año 2016 donde se registraron 23 pacientes.

[8] La edad de presentación más común de presentación al momento del diagnóstico en los pacientes con hernia incisional fue en mayores de 60 años con un total de 85 pacientes.

\section{Bibliografía.}

1. Bancalero L. Patología Urgente, de las hernías de la pared abdominal Madrid; 2013.

2. Egea y Tatay. Hernias abdomialesinternas Chicago; 2010.

3. Fogoros R. VerywellHealth. [Online]; 2018. Available from: 


\section{Prevalencia y factores de riesgo de hernias incisionales en adultos en el Hospital Abel Gilbert Pontón desde enero del 2014 a diciembre del 2017}

Vol. 3, núm. 2., (2019)

Isaías Rafael Dávila León; Ana María Viteri Rojas; Jorge Luis Gaitan Jiménez; Karina Johana

Cevallos Cárdenas; Angélica Katherine Sánchez Alcántara

https://www.verywell.com/what-is-incisional-hernia-3157228.

4. Matzke G y Espil G. Rey Argent Radiol. [Online].; 2017. Available from: http://linkinghub.elsevier.com/retrieve/pii/S0048761916300278.

5. Paz y Varela. Hernias de la Región Inguino - crural Madrid; 2008.

6. Speranzini M. ABCD ArgBrasCirdig Sao Paulo. [Online].; 2010. Available from: http://www.scielo.br/scielo.php?script=sci_abstract\&pid=S0102-

$\underline{67202010000400015 \& \operatorname{lng}=\mathrm{e} \& \& n r m=i s o \& t \operatorname{lng}=\mathrm{en}}$.

7. Yanchouchy-Chouillard E. DigSurg. [Online].; 2003. Available from: https://www.karger.com/Article/FullText/68850.

8. Cochrane. Procedimientos de cirugía abierta para la hernia incisional. [Online].; 2018. Available from: /es/CD006438/procedimientos-de-cirugia-abierta-para-la-hernia-incisional.

9. Cirugía General. Hernias de pared abdominal. [Online].; 2018. Available from: http://www.cirugiageneral.net/hernias.html.

10. WEBMD. WebMD. [Online].; 2018. Available from: https://www.webmd.com/digestivedisorders/tc/common-types-of-hernias-incisional-hernia.

11. Mountsiani Health. Incisional Hernia - Risk Factors \& Treatment. [Online].; 2018. Available from:

http://www.mountsinai.org/care/surgery/services/generalsurgery/conditions/hernia/incisional-hernia.

12. UW Medicine. Repair of Ventral/ Incisional Hernias. [Online].; 2018. Available from: Disponible en: https://www.uwmedicine.org/health-library/pages/repair-of-ventral-incisionalhernias.aspx.

13. WEB MD. Web Md. [Online].; 2018. Available from: https://www.webmd.com/digestivedisorders/understanding-hernia-basics.

14. Matzke G. Karger. [Online].; 2003. Available from: https://www.karger.com/Article/FullText/68850. 